\title{
Clinical Benefit of Gastric Staple Line Reinforcement (SLR) in Gastrointestinal Surgery: a Meta-analysis
}

\author{
Scott A. Shikora ${ }^{1}$ - Christine B. Mahoney ${ }^{2}$
}

Published online: 13 May 2015

(C) Springer Science+Business Media New York 2015

\begin{abstract}
Background The objective of this study was to assess whether the use of staple line reinforcement (SLR) reduces staple line complications (SLC). Mechanical staple lines are essential for gastrointestinal surgery such as bariatric surgery. However, SLC, such as bleeding and leakage, still occur. The purposes of this study were to provide quantitative evidence on the relative efficacy of gastric SLR and to compare the rates of effectiveness of three commonly used methods.

Methods A search of the medical literature in English language journals identified studies from Jan 1, 2000, to Dec 31, 2013, using the following reinforcement types: (1) no reinforcement, (2) oversewing, (3) a biocompatible glycolide copolymer, and (4) bovine pericardium after gastric bypasses and sleeve gastrectomies. Types of reinforcement were compared using a random-effects model.

Results This meta-analysis reviewed 16,967 articles, extracting data on 56,309 patients concerning leak and 41, 864 patients concerning bleeding. Over $40 \%$ of patients had no reinforcement, resulting in the highest leak rate $(2.75 \%)$
\end{abstract}

Mechanical staple lines are essential to gastrointestinal surgery such as bariatric surgery. Staple line complications (SLC) such as bleeding or leakage can occur. There is controversy whether staple line reinforcement can reduce SLC. This Meta-analysis demonstrated that SLR does in fact significantly reduce SLC but the various methods are not equivalent.

Scott A. Shikora

sshikora@partners.org

Christine B. Mahoney

Christine.mahoney@mnsu.edu

1 Department of Surgery, Brigham and Women's Hospital, 75 Francis Street, ASB II, Boston, MA 02115, USA

2 Mankato College of Business, Minnesota State University, Mankato, MN 56001, USA and bleed rate $(3.45 \%)$. Overall, reinforcing with bovine pericardium had the lowest leak (1.28 \%) and bleed (1.23\%) rates. Suture oversewing was better than no reinforcement but not as effective as bovine pericardium for leak $(2.45 \%)$ and bleed (2.69\%) rates. Buttressing with a biocompatible glycolide copolymer resulted in the second highest leak rate $(2.61 \%)$ and a bleed rate of $2.48 \%$ but had significantly lower bleed rates than no reinforcement.

Conclusions SLR provided superior results for patients compared to no reinforcement for reducing SLC. Buttressing with bovine pericardium resulted in the most favorable outcomes. The effectiveness of different methods used to reinforce the staple line in gastric surgery does not appear to be equal.

Keywords Staple lines · Staple line leak · Staple line bleed · Staple line oversewing $\cdot$ Staple line buttress .

Bioabsorbable glycolide copolymer · Bovine pericardium

\section{Introduction}

Since first being introduced to the USA by Ravitch et al. [1], mechanical stapling devices have long been essential for gastrointestinal surgery. Furthermore, without the availability of these instruments, gastrointestinal surgery could not be performed as routinely as it is. Stapling devices can create transections and anastomoses quickly, safely, and with minimal, if any, bleeding or spillage. They may also result in fewer postoperative leaks and bleeds. Mechanical stapling devices provide a tremendous advance from the traditional use of scissors, clamps, and suture. The introduction of stapling devices into gastrointestinal surgery has contributed to the reduction in operating time and perioperative complications, both of which simultaneously decrease the cost of the procedure and improve the outcomes for the individual patient. 
Currently, the linear cutting stapler, a device that creates two staple lines each comprised of three rows of individual staples, and simultaneously cuts the tissue between the two, is employed for nearly all gastric partitions, gastrointestinal resections, and the majority of gastrointestinal hollow organ anastomoses.

In the specialty of bariatric surgery, the safe and precise creation of gastric bypass pouches, anastomoses, and gastric sleeves is necessary for effective long-term results. The current stapler designs are relatively simple to use and reliable, creating durable staple lines nearly every time. In addition, surgical staplers are responsible in part for the current popularity of bariatric surgery since the procedures have become increasingly safe and efficacious [2-6]. However, despite the remarkable reliability of the current generation of surgical staplers, staple line complications do still occur and can result in significant morbidity and mortality [7].

Serious staple line complications include hemorrhage and leak. In bariatric surgery, while the incidence of both complications is relatively low (1-6\% for bleeding, $1-6 \%$ for leakage), the results can be both clinically devastating for the patient and expensive for the facility [8-14]. Therefore, any maneuvers that can reduce the likelihood of these complications will be of great interest to gastrointestinal and bariatric surgeons.

At present, there are several options for reinforcing gastric staple lines. Surgeons can oversew the staple line with suture or buttress the staple line with either synthetic polymers or biologic tissue-buttressing materials. There are many surgeons who chose not to reinforce staple lines either for concern over the cost and/or the lack of conclusive published benefit.

To date, there have been numerous small studies that have reported on the benefit or lack of benefit of these maneuvers [15-20]. The majority of these studies are small in size and provide inadequate information to conclude which method, if any, truly reduces staple line bleeding or leakage. Additionally, some of the meta-analyses published do not use appropriate statistical methods but instead rely on simplistic and incorrect statistical techniques. For example, some published metaanalyses and systematic reviews with quantitative analysis combine patients from all of the chosen studies. To arrive at a conclusion, the patients are added together as if they were in one study. The studies were not weighted by size or probability (as they must be), thereby providing inaccurate results [20, 21]. A true meta-analysis does not just add all the patients together as if they were in one big study but calculates a value for each study based on size and variance. Others did not include a full evaluation of the current literature. For these reasons, there is unfortunately no consensus drawn as to the benefit or lack thereof of staple line reinforcement. Therefore, there remains a need for more extensive information to accurately determine if staple line reinforcement is beneficial. The purpose of this study was to use a meta-analysis to provide quantitative evidence on the relative efficacy of staple line reinforcement.

\section{Methods}

\section{Data Sources and Searches}

The study design was guided by the use of the Preferred Reporting Items for Systematic Reviews and Meta-analyses statement (PRISMA) [22, 23].

A comprehensive search was done of the medical literature, querying studies and abstracts published in English language journals from Jan 1, 2000, to Dec 31, 2013. Published studies were identified by searching MEDLINE, ClinicalTrials.gov, the Cochrane Library, and Google Scholar. Additionally, bibliographies of all retrieved articles were searched for studies not found in the standard search [24]. Searches were constrained to peer-reviewed, refereed studies and abstracts published in English. Abstracts from peer-reviewed proceedings were included to address the "file drawer," or publication bias problem, a common criticism of meta-analyses [24-27].

This study conforms to the standard definition of a systematic review, "A systematic review collates all empirical evidence regarding a particular research question, the key characteristics being:

- a clearly stated set of objectives (pre-defined eligibility criteria for studies);

- an explicit, reproducible methodology;

- a systematic search that attempts to identify all studies that would meet the eligibility criteria;

- an assessment of the validity of the findings of the included studies, e.g. assessment of risk of bias;

- a systematic presentation, and synthesis, of the characteristics and findings of the included studies. Many systematic reviews contain meta-analyses." [28].

\section{Study Selection and Criteria}

The keywords used were "sleeve gastrectomy," "vertical gastrectomy," "roux-en-Y," and "gastric bypass." The resulting abstracts were reviewed for specific exclusion criteria: metaanalyses, reviews, letters to the editor, case reports, studies with less than five patients, animal studies, non-English language publications, open surgery, bariatric surgeries other than sleeve gastrectomy or gastric bypass, duplicate studies, population-level studies (they provide no staple-line reinforcement detail), data on staple line reinforcement or lack thereof not available, complications not reported, and complications not available by type of reinforcement. Any studies that reported only on a very high-risk group, e.g., all revision surgeries, were also excluded. Reinforcement types included in the analysis were (1) no reinforcement, (2) oversewing and two types of buttress material, (3) a biocompatible glycolide copolymer, and (4) bovine pericardium. For studies where a 
group published the same data more than once and repeated reports of a series, care was taken to assure that patients were included in the analysis only once.

The inclusion of non-randomized or observational studies was necessary because the majority of the bariatric surgery studies are not randomized trials. The most current accepted methods were utilized to assess the quality of these observational studies [29, 30]. The use of these non-randomized studies (NRS) is useful in many fields, such as bariatric surgery, when "To provide evidence of the effects (benefit or harm) of interventions that cannot be randomized, or which are extremely unlikely to be studied in randomized trials" [31].

\section{Data Extraction}

All outcomes, complications, and results reported were extracted from all included studies and abstracts. All patient demographics reported were also extracted. For any inconsistencies in data presentation in the studies, study authors were contacted via email to clarify the data in question. Data was extracted separately by surgical procedure and type of buttress used. Data was extracted from both randomized controlled trials and observational studies.

\section{Statistical Analysis}

For this analysis, "no reinforcement" was compared to "suture reinforcement" and to buttressing with the two most commonly used buttresses, bovine pericardium (Peristrips Dry ${ }^{\circledR} /$ Peristrips Dry with Veritas ${ }^{\circledR}$ Baxter Healthcare, Deerfield, IL), and a biocompatible glycolide copolymer buttress (Seamguard ${ }^{\circledR}$, W.L. Gore \& Associates, Inc, Flagstaff, AZ). Each type of reinforcement was compared to every other type, resulting in six comparisons. Types of reinforcement were compared using a random-effects model; statistical analyses were performed using Comprehensive Meta-analysis, 2.2.064 (Biostat, Englewood, NJ). This includes calculation of effect sizes, heterogeneity tests, meta-analyses, funnel plots, and forest plots using the software's randomeffects models.

There are two stages in a meta-analysis: the results for each study are calculated; then, a pooled average of those results is calculated. First, data is extracted from each individual study and the result for that study is calculated (the point estimate or summary statistic), with an estimate of the chance variation we would expect with that study (a confidence interval). Then all the point estimates for each study are pooled, being weighted by size and variance [28]. The study size and variation from each study must be incorporated into the analysis if the results are to be accurate and generalizable. Studies that call themselves a "meta-analysis," and definitely are not a meta-analysis, result in readers becoming distrustful of all meta-analyses.

\section{Our hypotheses were}

(1) Does a significant difference in adverse patient outcomes result from the uses of different types of reinforcement?

(2) What is the magnitude of the difference in adverse patient outcomes across studies?

Accumulating data from many studies performed by independent researchers means it is not likely that the surgeries within the studies are performed similarly. In the case of the bariatric surgeries of interest here, there are differences in surgeons' experience and techniques used, surgical protocols, post-surgical care and protocols, resources, and patient populations. The effect size can be greater or smaller, for example, if the study participants are older and less healthy overall, if the surgeon's skill and experience differ, or if institutional resources differ. These differences all impact the results of the studies, requiring the use of a random-effects model for analysis. The random effects also allow us to generalize the results to situations that may differ from the situation of the particular studies.

\section{Results}

A total of 16,967 articles were screened using their abstracts; 15,348 were rejected based on our exclusion criteria. The remaining 1619 studies needed further analysis to determine inclusion. A further 1324 studies were rejected based on this analysis, and 295 studies were retained for the data extraction. The most common reasons for rejecting studies were no data for study (350), not enough staple line reinforcement detail to assign to group (228), and different types of staple line reinforcement used with complication data pooled (140) (Fig. 1).

Data was extracted on 56,309 patients for leak and 41,864 patients for bleeding; there were 66,727 unique patients in the analysis. These patients were included in 516 study arms, 301 for leak and 215 for bleed. As depicted in Table 1, over $40 \%$ of the patients did not have staple line reinforcement of any type (46\% in the leak data and $43 \%$ in the bleeding data). Oversewing with suture was the next most common followed by the use of bovine pericardium, then the biocompatible glycolide copolymer.

As demonstrated in Table 2, the incidence of staple line leaks was highest for the patients who had no buttressing $(2.75 \%)$ and second highest was with the biocompatible glycolide copolymer $(2.61 \%)$. The lowest incidence of leak was seen with the bovine pericardial buttressing $(1.28 \%)$. Suture oversewing resulted in an intermediate outcome $(2.45 \%)$. Differences in leak rates were significant between none and bovine pericardium, oversew and bovine pericardium, and bovine pericardium and glycolide copolymer. 
Fig. 1 Study attrition diagram

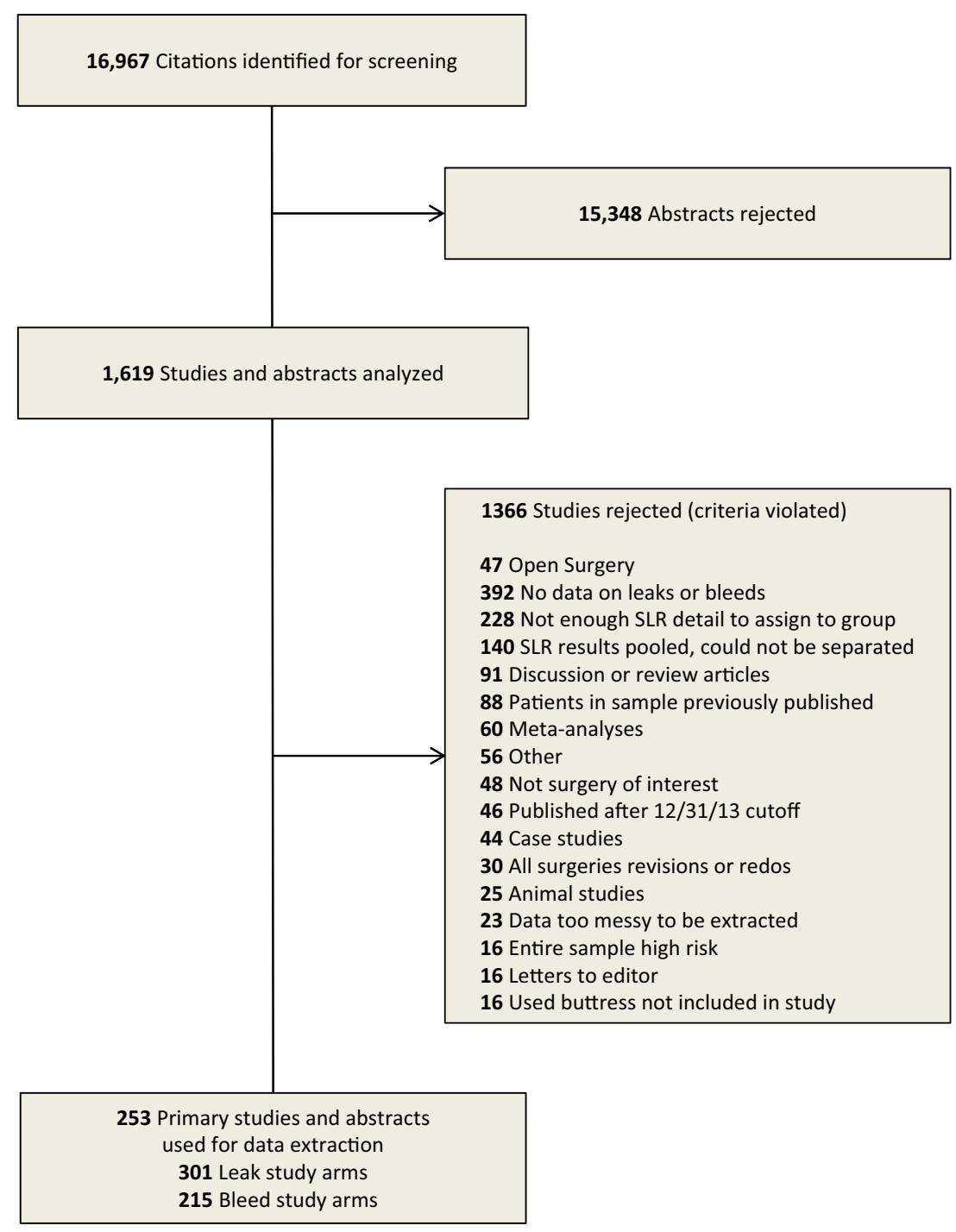

Similar results were seen with bleeding (Table 3). The highest incidence of bleeding, $3.45 \%$, was seen when there was no reinforcement of the staple lines and the lowest incidence was reported for the staple lines buttressed with bovine pericardium, $1.23 \%$. The use of a glycolide copolymer or suture oversewing resulted in intermediate rates of bleeding, 2.48 and $2.69 \%$, respectively. In fact, all reinforcement

Table 1 Use of each reinforcement option

\begin{tabular}{lccc}
\hline Buttress material & \multicolumn{2}{l}{ Number of patients (\%) } \\
\cline { 2 - 4 } & Leak & Bleed & Total \\
\hline None & $26,023(46.2)$ & $17,808(42.5)$ & $43,831(44.6)$ \\
Oversuture & $19,755(35.1)$ & $14,368(34.3)$ & $34,123(34.8)$ \\
Bovine pericardium & $6838(12.1)$ & $6759(16.1)$ & $13,597(13.9)$ \\
Glycolide copolymer & $3693(6.6)$ & $2929(7.0)$ & $6622(6.7)$ \\
\hline
\end{tabular}

options except suture oversewing resulted in statistically significantly less bleeding than doing nothing $(P<0.05)$. The differences in bleed rates were significant between none and bovine pericardium, oversew and bovine pericardium, bovine pericardium and biocompatible glycolide copolymer, and none and biocompatible glycolide copolymer.

Table 2 Leak rate by reinforcement type (sleeve and gastric bypass)

\begin{tabular}{lclc}
\hline Buttress material & $\begin{array}{l}\text { Number of } \\
\text { study arms }\end{array}$ & $\begin{array}{l}\text { Event rate } \\
\text { (high to low) }\end{array}$ & $\begin{array}{l}\text { Number of } \\
\text { patients }\end{array}$ \\
\hline None & 116 & $2.75 \%$ & 26,023 \\
Glycolide copolymer & 52 & $2.61 \%$ & 3693 \\
Oversuture & 92 & $2.45 \%$ & 19,755 \\
Bovine pericardium & 41 & $1.28 \%$ & 6838 \\
\hline
\end{tabular}

Bovine pericardium versus none $-p<0.001$; bovine pericardium versus oversuture $-p<0.001$; bovine pericardium versus glycolide copolymer$p<0.01$ 
Table 3 Bleed rate by reinforcement type (sleeve and gastric bypass)

\begin{tabular}{lllc}
\hline Buttress material & $\begin{array}{l}\text { Number of } \\
\text { study arms }\end{array}$ & $\begin{array}{l}\text { Event rate } \\
\text { (high to low) }\end{array}$ & $\begin{array}{l}\text { Number } \\
\text { of patients }\end{array}$ \\
\hline None & 83 & $3.45 \%$ & 17,808 \\
Oversuture & 58 & $2.69 \%$ & 14,368 \\
Glycolide copolymer & 44 & $2.48 \%$ & 2929 \\
Bovine pericardium & 30 & $1.23 \%$ & 6759 \\
\hline
\end{tabular}

Bovine pericardium versus none $-p<0.001$; bovine pericardium versus glycolide copolymer $-p<0.01$; bovine pericardium versus oversuture$p \leq 0.05$; glycolide copolymer versus none $-p<0.01$

The type of operative procedure had no effect on the results. The outcomes reported above were consistent for sleeve gastrectomy and gastric bypass. Leak and bleed rates for sleeve gastrectomy are reported in Tables 4 and 5. Once again, doing nothing had the highest leak and bleed rates and bovine pericardium, the lowest. The significance of differences between reinforcement methods is shown. Leak and bleed rates for gastric bypass are reported in Tables 6 and 7; they follow similar patterns.

\section{Discussion}

The concept of staple line reinforcement is not a new one. Despite the relative safety and reliability of the stapling devices, complications can and do occur. Both leaks and bleeds have been reported to occur from 1 to $6 \%[4,5,8-14]$. In this meta-analysis, unreinforced staple lines had an incidence of leakage of $2.75 \%$ and a bleeding rate of $3.45 \%$. The outcomes of these complications can range from relatively minor sequelae to extremely severe and life-threatening events. Therefore, it has long been the desire of gastrointestinal surgeons to consider employing any maneuver that could potentially reduce these risks.

Many different reinforcement options have and are being used. In a recent survey of experienced sleeve gastrectomy surgeons for the International Sleeve Gastrectomy Expert Panel Consensus Statement [32], Rosenthal et al. reported that $100 \%$ of participants agreed that reinforcement reduced

Table 4 Leak rate by reinforcement type for sleeve gastrectomy

\begin{tabular}{llll}
\hline Buttress material & $\begin{array}{l}\text { Number of } \\
\text { study arms }\end{array}$ & $\begin{array}{l}\text { Event rate } \\
\text { (high to low) }\end{array}$ & $\begin{array}{l}\text { Number of } \\
\text { patients }\end{array}$ \\
\hline None & 35 & $3.27 \%$ & 3958 \\
Glycolide copolymer & 33 & $3.25 \%$ & 1850 \\
Oversuture & 47 & $2.70 \%$ & 6141 \\
Bovine pericardium & 16 & $1.83 \%$ & 1678 \\
\hline
\end{tabular}

Bovine pericardium versus none $-p<0.01$; bovine pericardium versus oversuture $-p<0.05$; bovine pericardium versus glycolide copolymer$p<0.01$
Table 5 Bleed rate by reinforcement type for sleeve gastrectomy

\begin{tabular}{llll}
\hline Buttress material & $\begin{array}{l}\text { Number } \\
\text { of study arms }\end{array}$ & $\begin{array}{l}\text { Event rate } \\
\text { (high to low) }\end{array}$ & $\begin{array}{l}\text { Number } \\
\text { of patients }\end{array}$ \\
\hline None & 25 & $4.94 \%$ & 2865 \\
Oversuture & 33 & $2.41 \%$ & 4682 \\
Glycolide copolymer & 28 & $2.09 \%$ & 1997 \\
Bovine pericardium & 14 & $1.16 \%$ & 1632 \\
\hline
\end{tabular}

Bovine pericardium versus none $-p<0.001$; oversuture versus none$p<0.001$; glycolide coploymer versus none $-p<0.001$; bovine pericardium versus oversuture $-p<0.05$; bovine pericardium versus glycolide copolymer $-p<0.05$

bleeding. Seventy-seven percent thought it was acceptable to buttress the staple line and $95 \%$ supported oversewing. However, in that group of recognized "sleeve experts," only $63 \%$ suture-reinforced the staple line and only $21 \%$ used a buttressing material. The discrepancy between supporting its use and actually using it may lie in the concern for the perceived lack of published results and the "high" cost of buttressing. It is also interesting to point out that the greater incidence of both suture reinforcement and buttressing seen in the Expert Panel Consensus Statement versus this metaanalysis may suggest either that the prevalence of staple line reinforcement is increasing or that the experts are more likely to reinforce staple lines than less-experienced surgeons.

Overall, oversewing of gastrointestinal staple lines is likely the most commonly performed method for reinforcement. Consistent with the prevalence reported in the Expert Panel Consensus Statement [32], we found that it was employed nearly twice as often as the next most common, buttressing with bovine pericardium. Oversewing is clearly the least costly in terms of monetary resources but may dramatically increase surgery time, which is also costly. However, for those who suture oversew, there is no consensus as to which suture material (absorbable vs. nonabsorbable) or type of sewing technique (baseball stitch, simple oversewing, locking, imbricating, etc.) is best. In addition, while some surgeons oversew the entire staple line, others only selected regions of the staple line. Oversewing a staple line has not unanimously been shown to be beneficial and might, in fact, lead to a greater

Table 6 Leak rate by reinforcement type for gastric bypass

\begin{tabular}{llll}
\hline Buttress material & $\begin{array}{l}\text { Number of } \\
\text { study arms }\end{array}$ & $\begin{array}{l}\text { Event rate } \\
\text { (high to low) }\end{array}$ & $\begin{array}{l}\text { Number of } \\
\text { patients }\end{array}$ \\
\hline None & 81 & $2.60 \%$ & 22,065 \\
Oversuture & 45 & $2.44 \%$ & 13,614 \\
Glycolide copolymer & 19 & $1.88 \%$ & 1843 \\
Bovine pericardium & 25 & $1.00 \%$ & 5160 \\
\hline
\end{tabular}

Bovine pericardium versus none $-p<0.001$; bovine pericardium versus oversuture $-p<0.001$; bovine pericardium versus glycolide copolymer$p<0.05$ 
Table 7 Bleed rate by reinforcement type for gastric bypass

\begin{tabular}{lllc}
\hline Buttress material & $\begin{array}{l}\text { Number of } \\
\text { study arms }\end{array}$ & $\begin{array}{l}\text { Event rate } \\
\text { (high to low) }\end{array}$ & $\begin{array}{l}\text { Number of } \\
\text { patients }\end{array}$ \\
\hline None & 58 & $3.13 \%$ & 14,943 \\
Oversuture & 25 & $3.10 \%$ & 9686 \\
Glycolide copolymer & 16 & $3.02 \%$ & 932 \\
Bovine pericardium & 16 & $1.28 \%$ & 5127 \\
\hline
\end{tabular}

Bovine pericardium versus none $-p<0.01$; bovine pericardium versus oversuture $-p<0.01$; bovine pericardium versus glycolide copolymer$p<0.05$

incidence of staple line complications as a consequence of ischemia or tissue tearing [33]. In this study, it was beneficial when compared to doing nothing but not as efficacious as buttressing with bovine pericardium.

Staple line buttressing has been shown in several publications to decrease bleeding [17, 18, 34-36], increase staple line burst pressure [37-39], and possibly even reduce leak rates $[16,40,41]$. However, as stated above, its use is not widespread. This may be due in part to the concern of added cost. However, the cost of the product is only one factor when determining total cost. To better assess the total financial cost, one must also take into account other factors such as operating room time and improvements in outcome (i.e., reduction of complications, the need for other resources, reduction of hospital length of stay). For example, while several studies have confirmed that buttressing materials reduce intraoperative bleeding, the difference in estimated blood loss was clinically insignificant. However, in some studies, with the use of buttressing materials, the reduction in costly operating room time was greater than $30 \mathrm{~min}$ per case [17,34] and the length of hospitalization was reduced almost a full day [35].

The reduction in the incidence of gastrointestinal leakage would be of even greater significance. However, proving that an intervention decreases leakage is extremely challenging. Since the basal leak rate is rather low, powering a study sufficiently to result in statistically significant differences would require large numbers of patients and likely impossible to perform. Yet despite the relatively low incidence, leaks are often highly clinically significant and can result in prolonged hospitalization, critical illness, sepsis, debilitation, and even death. In fact, a recent study by Zambelli-Weiner et al. has estimated that the mean hospital charge for a leak after sleeve gastrectomy was $\$ 137,417$ and over $\$ 400,000$ if it resulted in sepsis [42]. A reduction in the leak rate of $0.5 \%$, over 1000 patients, would result in a savings of over $\$ 687,085$ at this rate. The cost of a leak can even go much higher. Therefore, the reduction in operating time and the possible reduction of costly complications suggest that the additional costs for the use of buttressing may be justified.

This study is the largest meta-analysis to date evaluating the outcomes for staple line reinforcement. Data was extracted and pooled from 56,309 patients for leak and 41,864 patients for bleeding. The results of this meta-analysis convincingly demonstrate that reinforcing gastrointestinal staple lines reduces complications such as leakage and bleeding. Additionally, the type of reinforcement mattered. Bovine pericardium seemed to be superior for preventing leakage and bleeding to both suture reinforcement and a biocompatible glycolide copolymer buttress. Smaller studies reported conflicting results. Our results are consistent with the recent meta-analysis performed exclusively for sleeve gastrectomy by Choi et al. [43]. They analyzed the outcomes for 1345 patients and found that staple line reinforcement with a buttress significantly reduced the incidence of bleeding, leakage, and overall complications. Oversewing showed no such advantage and, in fact, was found to increase the bleeding risk. In a meta-analysis of 180 laparoscopic gastric bypass patients extracted from 3 studies, Sajjid et al. also demonstrated similar results [44]. Although the number of patients included in the study was quite small, they demonstrated that patients with buttressed staple lines had statistically significantly shorter operating times $(P<0.01)$, fewer postoperative complications $(P<0.01)$, and fewer leaks $(P<0.03)$ than patients without reinforcement. No difference in staple line bleeding was demonstrated, but the unreinforced patients required more surgical clips to control bleeding.

In contrast, Giannopoulos et al. performed a meta-analysis of bariatric patients and observed contradictory results [45]. Analyzing the data from a pooled sample of 3299 patients, they concluded that there was no benefit to buttressing over the control. Staple line oversewing was not included in their analysis. Similarly, in a systemic review of 4881 patients undergoing sleeve gastrectomy, Knapps et al. [46] found no significant differences in leak rates, mortality, and overall morbidity between reinforced and unreinforced patients. However, they did not do subset analysis of the data to see if there were differences based on the type of reinforcement employed. Parikh et al. did a systematic review and metaanalysis of sleeve gastrectomy papers. Six thousand five hundred seventy-eight patients were identified that had buttressed staple lines. They report no benefit of buttressing for reducing leaks [47]. However, their study had several limitations. Firstly, they only included papers published from 2005 to 2011, thereby excluding any data published after 2011. Sleeve popularity and case volume have taken off since 2011. Secondly, $56 \%$ of patients had biocompatible glycolide copolymer buttresses with either Seamguard or Duet. There is not enough data on Duet to include it in an analysis, and the product was subsequently withdrawn by the manufacturer from the market.

Additionally, most of these studies suffer from being statistically underpowered. Staple line leaks and bleeds occur at such low rates that an extremely large number of patients must be included to achieve statistically significant differences. 
One question raised by this study is why different buttressing materials responded differently. Peristrips Dry ${ }^{\circledR /}$ Peristrips Dry with Veritas ${ }^{\circledR}$ is derived from bovine pericardium. In contrast, the biocompatible glycolide copolymer buttress, Seamguard ${ }^{\circledR}$, is synthesized from polyglycolic acid and trimethylene carbonate. Therefore, it is very possible that they differ in performance due to their different thickness and possibly even their different makeup.

Buttressing is thought to reduce bleeding and leaks by adding thickness to the staple line and by distributing the tension of any individual staple across the length of the reinforcement strip. All of the available products add thickness to the stapled tissue. The better results demonstrated with bovine pericardium over an absorbable buttress may be solely due to its increased thickness ( 1 vs. $0.5 \mathrm{~mm}$ ). However, in a hemostasis animal model, Spector et al. [48] reported that a collagen matrix biologic buttress was superior to a synthetic buttress for promoting hemostasis and in fact performed as well as a conventional hemostasis product.

Unfortunately, it is still unclear why the bovine pericardial buttress outperformed the bioabsorbable glycolide copolymer for reducing the likelihood of leakage. In stark contrast, Gagner et al. [20] recently performed their own systematic review and found conflicting results. They analyzed 88 publications consisting of 8920 patients. They found that staple line reinforcement was beneficial but the use of a biocompatible glycolide copolymer buttress had a significantly lower leak rate than the use of bovine pericardium (1.09 vs. $3.3 \%$, $P<0.0006)$. However, in their review, only 665 patients with bovine pericardium and 1446 with biocompatible buttress were included compared to 5160 patients with bovine pericardium and 1850 with bioabsorbable buttress in this review. Clearly, the dramatically smaller number of patients with bovine pericardium could have skewed the results. In addition, as only 88 publications were used for their systematic review compared with 295 publications included in the current metaanalysis, were those 88 papers truly representative of the performance of the 2 products? Further investigation is necessary to confirm whether the superiority of one type of buttress versus another truly exists and, if so, what is the mechanism for the difference?

Like all meta-analyses, this one suffered from several limitations that could have affected the reported outcomes. Firstly, the meta-analysis was not limited to randomized controlled trials, because very few exist in the bariatric surgery literature. Therefore, the studies used are almost all retrospective. Many of the studies were small-under 100 patients. Secondly, the bariatric surgery literature is quite inconsistent in the use of terminology for techniques, materials, and definition of outcomes. Thirdly, there was no uniformity in the conduct of the surgeries. For example, the choice of stapler, staple cartridge, bougie size, oversewing technique, buttress material, etc. was solely selected by the surgeon and varied greatly from study to study. Additionally, there is also no consensus definition for labeling a bleed or a leak. Some investigators only reported a bleeding episode if it required transfusion or emergency surgery. Others based the definition on a predetermined amount of blood loss or drop in hemoglobin. Similarly, a leak can be a radiographic diagnosis or a clinical one. Some programs perform routine postoperative radiographic studies that will find very small non-clinical leaks while programs that do not perform routine postoperative X-rays will only report clinical leaks. The location of the leak from a gastric bypass procedure may be difficult to define. Therefore, a distal pouch leak could be from the linear staple line, the anastomosis, or both. Anastomotic leaks would obviously not be influenced by whether a linear staple line is reinforced or not but could be included in the incidence of leaks reported in the studies. As explained in the "Methods" section, this was addressed statistically with the use of the random-effects method. Furthermore, one would assume that the differences discovered in the definitions, surgical techniques, and the surgeons' choices would be evenly distributed across all four groups.

Lastly, the overwhelming majority of the patients in this study were morbidly obese patients undergoing bariatric surgery. While it would be reasonable to conclude that the results would be similar for non-morbidly obese individuals undergoing other gastrointestinal surgeries, to be valid, that premise would need to be evaluated independently.

\section{Conclusion}

This large meta-analysis demonstrated that in a population predominantly comprised of morbidly obese bariatric patients, staple line reinforcement significantly reduced the likelihood of staple line leaking and bleeding. Furthermore, a biologic buttress outperformed both oversewing with suture and a synthetic buttress.

Acknowledgments This study was supported by a grant from Baxter Healthcare.

Ethical Statement Ethical approval and informed consent do not apply to this article.

\section{References}

1. Ravitch MM, Steichen FM. Staple suturing in the gastrointestinal tract. Ann Surg. 1972;175:815-36.

2. The Longitudinal Assessment of Bariatric Surgery (LABS) Consortium. Perioperative safety in the longitudinal assessment of bariatric surgery. N Engl J Med. 2009;361:445-54.

3. Buchwald H, Estok R, Fahrbach K, et al. Weight and type 2 diabetes after bariatric surgery: systemic review and meta-analysis. Am J Med. 2009;122:248-56. 
4. Chang SH, Stoll CR, Song J, et al. The effectiveness and risks of bariatric surgery: an updated systematic review and meta-analysis, 2003-2012. JAMA Surg. 2014;149:275-87.

5. Banka G, Woodard G, Hernandez-Boussard T, et al. Laparoscopic vs open gastric bypass surgery: differences in patient demographics, safety, and outcomes. Arch of Surg. 2012;147:550-6.

6. Benotti P, Wood GC, Winegar DA, et al. Risk factors associated with mortality after Roux-en-Y gastric bypass surgery. Annals of Surg. 2014;259:123-30.

7. Wassner JD, Yohai E, Heimlich H. Complications associated with the use of gastrointestinal stapling devices. Surgery. 1977;82:393-9.

8. Frezza EE, Reddy S, Gee LL, et al. Complications after sleeve gastrectomy for morbid obesity. Obes Surg. 2009;19:684-7.

9. Burgos AM, Braghetto I, Csendes A, et al. Gastric leak after laparoscopic sleeve gastrectomy. Obes Surg. 2009;19:1672-7.

10. Aggarwal S, Kini SU, Herron DM. Laparoscopic sleeve gastrectomy for morbid obesity: a review. Surg Obes Relat Dis. 2007;3:189-94

11. Shikora SA, Kim JJ, Tarnoff ME, et al. Laparoscopic Roux-Y gastric bypass: results and learning curve of a high volume academic program. Arch Surg. 2005;140:362-7.

12. Schauer PR, Ikramuddin S, Gourash W, et al. Outcomes after laparoscopic Roux-en-Y gastric bypass for morbid obesity. Ann Surg. 2000;232:515-29.

13. Higa KD, Boone KB, Ho T. Complications of the laparoscopic Roux-en-Y gastric bypass: 1040 patients - what have we learned. Obes Surg. 2000;10:509-13.

14. Nguyen NT, Longoria M, Chalifoux S, et al. Early gastrointestinal hemorrhage after laparoscopic gastric bypass. Obes Surg. 2004;14: 1308-12.

15. Shikora SA, Kim JJ, Tarnoff ME. Comparison of permanent and nonpermanent staple line buttressing materials for linear gastric staple lines during laparoscopic Roux-en-Y gastric bypass. Surg Obes Relat Dis. 2008;4:729-34.

16. Chen B, Kiriakopoulos A, Tsakayannis D, et al. Reinforcement does not necessarily reduce the rate of staple line leaks after sleeve gastrectomy. A review of the literature and clinical experiences. Obes Surg. 2009;19:166-72.

17. Miller KA, Pump A. Use of a bioabsorbable staple reinforcement material in gastric bypass: a prospective randomized clinical trial. Surg Obes Relat Dis. 2007;3:417-21. Discussion 422.

18. Gagner M, Consten EC, Pomp A, et al. Decreased bleeding after laparoscopic sleeve gastrectomy with and without duodenal switch for morbid obesity using a buttressed absorbable polymer membrane. Obes Surg. 2004;14:1360-6.

19. Dapri G, Cadière GB, Himpens J. Reinforcing the staple line during laparoscopic sleeve gastrectomy: prospective randomized clinical study comparing three different techniques. Obes Surg. 2010;20: 462-7.

20. Gagner M, Buchwald JN. Comparison of laparoscopic sleeve gastrectomy leak rates in four staple-line reinforcement options: a systematic review. Surg Obes Relat Dis. 2014. doi:10.1016/j.soard. 2014.01.016.

21. Brethauer SA, Hammel JP, Schauer PR. Systematic review of sleeve gastrectomy as staging and primary bariatric procedure. Surg Obes Relat Dis. 2009;5:469-75.

22. Liberati A, Altman DG, Tetzlaff J, et al. The PRISMA statement for reporting systematic reviews and meta-analyses of studies that evaluate health care interventions: explanation and elaboration. Ann Int Med. 2009;151:W65-94.

23. Gleysteen JJ. Bariatric surgery literature in nonbariatric surgery journals: January 2009-June 2009. Surg Obes Relat Dis. 2010;3: 326-9.

24. Borenstein M, Hedges LV, Higgins PTH, et al. Introduction to meta-analysis. John Wiley \& Sons; 2011.
25. Field AP, Gillett R. How to do a meta-analysis. Br J Math Stat Psychol. 2010;63:665-94.

26. Chalmers TC, Levin H, Sacks HS, et all. Meta-analysis of clinical trials as a scientific discipline. I: Control of bias and comparison with large co-operative trials \& II: replicate variability and comparison of studies that agree and disagree. Stat Med. 1987;6:315-25 \& 733-44.

27. L'Abbe KA, Detsky AS, O'Rourke K. Meta-analysis in clinical research. Ann Intern Med. 1987;107:224-33.

28. Higgins, JPT. Green S. Cochrane handbook for systematic reviews of interventions version 5.1. 0 [updated March 2011]. The Cochrane Collaboration, 2014. www. cochrane-handbook. org.

29. Stroup DF, Berlin JA, Morton SC, et al. Meta-analysis of observational studies in epidemiology: a proposal for reporting. Metaanalysis of Observational Studies in Epidemiology (MOOSE) group. JAMA. 2000;283:2008-12.

30. Stang A. Critical evaluation of the Newcastle-Ottawa scale for the assessment of the quality of nonrandomized studies in meta-analyses. Eur J Epidemiol. 2010;25:603-5.

31. Reeves BC, Deeks JJ, Higgins JPT, et al. Chapter 13: Including non-randomized studies. In: Higgins JPT, Green S (editors), Cochrane handbook for systematic reviews of interventions version 5.1.0 (updated March 2011). The Cochrane Collaboration, 2011.

32. Rosenthal RJ, International Sleeve Gastrectomy Expert Panel. International Sleeve Gastrectomy Expert Panel Consensus Statement: best practice guidelines based on experience of $>12$, 000 cases. Surg Obes Relat Dis. 2012;8:8-19.

33. Baker RS, Foote J, Kemmeter P, et al. The science of stapling and leaks. Obes Surg. 2004;14:1290-8.

34. Angrisani L, Lorenzo M, Borrelli V, et al. The use of bovine pericardial strips on linear stapler to reduce extraluminal bleeding during laparoscopic gastric bypass: prospective randomized clinical trial. Obes Surg. 2004;14:1198-202.

35. Consten ECJ, Gagner M, Pomp A, et al. Decreased bleeding after laparoscopic sleeve gastrectomy with or without duodenal switch for morbid obesity using a stapled buttressed absorbable polymer membrane. Obes Surg. 2004;14:1360-6.

36. Nguyen NT, Longoria M, Welbourne S, et al. Glycolide copolymer staple-line reinforcement reduces staple site bleeding during laparoscopic gastric bypass. Arch Surg. 2005;140:773-8.

37. Pinheiro JS, Correa JL, Cohen RV, et al. Staple line reinforcement with new biomaterial increased burst pressure: an animal model. Surg Obes Relat Dis. 2006;2:397-9.

38. Arnold W, Shikora SA. A comparison of burst pressure between buttressed versus non-buttressed staple lines in an animal model. Obes Surg. 2005;15:164-71.

39. Downey DM, Harre JG, Dolan JP. Increased burst pressure in gastrointestinal staple-lines using reinforcement with a bioprosthetic material. Obes Surg. 2005;15:1379-83.

40. Shikora SA, Kim JJ, Tarnoff ME. Reinforcing gastric staple lines with bovine pericardial strips may decrease the likelihood of gastric leak after laparoscopic Roux-en-Y gastric bypass for morbid obesity. Obes Surg. 2003;13:37-44.

41. Al Hajj GN, Haddad J. Preventing staple-line leak in sleeve gastrectomy: reinforcement with bovine pericardium vs oversewing. Obes Surg. 2013;23:1915-21.

42. Zambelli-Weiner A, Brooks E, Brolin R, et al. Total charges for post-operative leak following laparoscopic sleeve gastrectomy. (Paper Presentation at Obesity Week 2013, Atlanta, Georgia, November 2013).

43. Choi YY, Bae J, Hur KY, et al. Reinforcing the staple line during laparoscopic sleeve gastrectomy: does it have advantages? A metaanalysis. Obes Surg. 2012;22:1206-13.

44. Sajjid MS, Khatri K, Singh K, et al. Use of staple line reinforcement in laparoscopic gastric bypass surgery: a meta-analysis. Surg Endosc. 2011;25:2884-91. 
45. Giannopoulos GA, Tzanakis NE, Rallis GE, et al. Staple line reinforcement in laparoscopic bariatric surgery; does it actually make a difference? A systemic review and meta-analysis. Surg Endosc. 2010;24:2782-8.

46. Knapps J, Ghanem M, Clements J, Merchant AM. A systematic review of staple line reinforcement in laparoscopic sleeve gastrectomy. JSLS. 2013;17:390-9.
47. Parikh M, Issa R, McCrillis A, et al. Surgical strategies that may decrease leak after laparoscopic sleeve gastrectomy. A systematic review and meta-analysis of 9991 cases. Ann Surg. 2013;257:231-7.

48. Spector D, Perry Z, Konobeck T, et al. Comparison of hemostatic properties between collagen and synthetic buttress materials used in staple line reinforcement in a swine splenic hemorrhage model. Surg Endosc. 2011;25:1148-52. 